\title{
VARIANTS OF EPIGROUPS AND PRIMARY CONJUGACY
}

\author{
MARIA BORRALHO AND MICHAEL KINYON*
}

\begin{abstract}
In a semigroup $S$ with fixed $c \in S$, one can construct a new semigroup $\left(S, \cdot{ }_{c}\right)$ called a variant by defining $x{ }_{c} y:=x c y$. Elements $a, b \in S$ are primarily conjugate if there exist $x, y \in S^{1}$ such that $a=x y, b=y x$. This coincides with the usual conjugacy in groups, but is not transitive in general semigroups. Araújo et al. proved that transitivity holds in a variety $\mathcal{W}$ of epigroups containing all completely regular semigroups and their variants, and asked if transitivity holds for all variants of semigroups in $\mathcal{W}$. We answer this affirmatively as part of a study of varieties and variants of epigroups.
\end{abstract}

\section{INTRODUCTION}

Let $S$ be a semigroup. Given $c \in S$ we can define a new binary operation ${ }_{c}$ on $S$ by

$$
a \cdot{ }_{c} b=a c b
$$

for all $a, b \in S$. The operation ${ }_{c}$ is clearly associative, and the semigroup $\left(S,{ }_{c}\right)$ and called the variant of $S$ at $c$ (see [20, and also [15, 16, 22, 24, 27, 30, 32, 36] and ([18, Chapter 13)).

Elements $a, b$ of a semigroup $S$ are said to be primarily conjugate, denoted $a \sim_{p} b$, if there exist $x, y \in S^{1}$ such that $a=x y$ and $b=y x$. Here as usual, $S^{1}$ denotes $S$ if $S$ is a monoid; otherwise $S^{1}=S \cup\{1\}$ where 1 is an adjoined identity element. Primary conjugacy is reflexive and symmetric, but it is not transitive in general. The transitive closure $\sim_{p}^{*}$ of $\sim_{p}$ can be considered to be a conjugacy relation in general semigroups $[19,25,26$. Primary conjugacy is transitive in groups (where it coincides with the usual notion of conjugacy) and free semigroups [28. To describe additional classes where primary conjugacy is known to be transitive, we must first recall the notion of epigroup.

An element $a$ of a semigroup $S$ is an epigroup element (also known as a group-bound element) if there exists a positive integer $n$ such that $a^{n}$ belongs to a subgroup of $S$, that is, the $\mathcal{H}$-class $H_{a^{n}}$ of $a^{n}$ is a group. The smallest such $n$ is the index of $a$. If $H_{a}$ itself is a group, that is, if $a$ has index 1 , then $a$ is said to be completely regular. If we let $e$ denote the identity element of $H_{a^{n}}$, then $a e$ is in $H_{a^{n}}$ and we define the pseudoinverse $a^{\prime}$ of $a$ by $a^{\prime}=(a e)^{-1}$, where $(a e)^{-1}$ denotes the inverse of ae in the group $H_{a^{n}}$ [35, (2.1)]. If every element of a semigroup is an epigroup element, then the semigroup itself is said to be an epigroup, and if every element is completely regular, then the semigroup is said to be completely regular. Every finite semigroup, and in fact every periodic semigroup, is an epigroup. Following Petrich and Reilly [33] for completely regular semigroups and Shevrin [34, 35] for epigroups, it is now customary to view an epigroup $(S, \cdot)$ as a unary semigroup $\left(S, \cdot{ }^{\prime}\right)$ where $x \mapsto x^{\prime}$ is the map sending each element to its pseudoinverse. We will make considerable use of the following identities which hold in all epigroups [35]:

$$
\begin{aligned}
x^{\prime} x x^{\prime} & =x^{\prime}, \\
x x^{\prime} & =x^{\prime} x, \\
x^{\prime \prime \prime} & =x^{\prime}, \\
x x^{\prime} x & =x^{\prime \prime}, \\
(x y)^{\prime} x & =x(y x)^{\prime}, \\
\left(x^{p}\right)^{\prime} & =\left(x^{\prime}\right)^{p},
\end{aligned}
$$

for any prime $p$.

1991 Mathematics Subject Classification. 20M07.

Key words and phrases. conjugacy, variants.

* Partially supported by Simons Foundation Collaboration Grant 359872 and by Fundaão para a Ciência e a Tecnologia (Portuguese Foundation for Science and Technology) grant PTDC/MAT-PUR/31174/2017. 
For each $n \in \mathbb{N}$, let $\mathcal{E}_{n}$ denote the variety (equational class) of all unary semigroups $\left(S, \cdot,^{\prime}\right)$ satisfying (1.2), (1.3) and $x^{n+1} x^{\prime}=x^{n}$. Each $\mathcal{E}_{n}$ is a variety of epigroups, and the inclusions $\mathcal{E}_{n} \subset \mathcal{E}_{n+1}$ hold for all $n$. Every finite semigroup, considered as an epigroup, is contained in some $\mathcal{E}_{n}$. $\mathcal{E}_{1}$ is the variety of completely regular semigroups.

The following observation will be useful later.

Lemma 1. For each $n \in \mathbb{N}$, the variety $\mathcal{E}_{n}$ is precisely the variety of unary semigroups satisfying (1.2), (1.3) and $x^{n-1} x^{\prime \prime}=x^{n}$.

Proof. If $S$ is an epigroup in $\mathcal{E}_{n}$, then $x^{n}=x^{n+1} x^{\prime}=x^{n-1} x x^{\prime} x=x^{n-1} x^{\prime \prime}$ using (1.3) and (1.5). Conversely, suppose $S$ satisfies (1.2), (1.3) and $x^{n-1} x^{\prime \prime}=x^{n}$. Then $x^{n+1} x^{\prime}=x^{n} x^{\prime \prime} x^{\prime}=x^{n-1} x^{\prime \prime} x^{\prime \prime} x^{\prime}=x^{n-1} x^{\prime \prime} x^{\prime} x^{\prime \prime}=$ $x^{n-1} x^{\prime \prime}=x^{n}$, using (1.3) in the third equality and (1.2) in the fourth equality.

Kudryavtseva 23] proved that the restriction of $\sim_{p}$ to the set of all completely regular elements of a semigroup is transitive. More recently, it was shown in [10] that $\sim_{p}$ is transitive in all variants of completely regular semigroups. Variants of completely regular semigroups are not, in general, completely regular themselves; for example, if a completely regular semigroup has a zero 0 , then the variant at 0 is a null semigroup, which is not even regular. This difficulty was circumvented in [10] by introducing the following class $\mathcal{W}$ of semigroups:

$$
S \in \mathcal{W} \quad \Longleftrightarrow \quad x y \text { is completely regular for all } x, y \in S .
$$

Equivalently $\mathcal{W}$ consists of all semigroups $S$ such that the subsemigroup $S^{2}=\{a b \mid a, b \in S\}$ is completely regular. The class $\mathcal{W}$ includes all completely regular semigroups and all null semigroups (semigroups satisfying $x y=u v$ for all $x, y, u, w)$. The following summarizes the relevant results of 10 .

\section{Proposition 2.}

(1) $\left(\left[10\right.\right.$, Prp. 4.14) $\mathcal{W}$ is the variety of epigroups in $\mathcal{E}_{2}$ satisfying the additional identity $(x y)^{\prime \prime}=x y$.

(2) ([10], Thm. 4.15) If $S$ is a epigroup in $\mathcal{W}$, then $\sim_{p}$ is transitive in $S$;

(3) (10], Thm. 4.17) Every variant of a completely regular semigroup is in $\mathcal{W}$;

(4) ([10], Cor. 4.18) If $S$ is a variant of a completely regular semigroup, then $\sim_{p}$ is transitive in $S$.

Part (2) of this proposition had more to it, comparing $\sim_{p}$ with other notions of conjugation. In the simplified form stated here, the result follows easily from Kudryavtseva's theorem [23]: if $a \sim_{p} b, b \sim_{p} c$, and $a \neq b \neq c \neq a$, then there exist $x, y, u, v \in S$ such that $a=x y, b=y x=u v$ and $c=v u$. Thus $a, b, c \in \mathcal{W}$ are completely regular, so $a \sim_{p} c$.

We can slightly improve Proposition 2(1) as follows.

Lemma 3. The variety $\mathcal{W}$ is precisely the variety of unary semigroups satisfying the identities (1.2), (1.3), (1.5), (1.7) (for $p=2$ ) and $(x y)^{\prime \prime}=x y$.

Proof. One implication follows from Proposition $2(1)$, so suppose $\left(S, \cdot,^{\prime}\right)$ is a unary semigroup satisfying the identities listed in the lemma. Then for all $x \in S$,

$$
\begin{gathered}
x^{3} x^{\prime} \stackrel{\text { (1.2) }}{=} x^{3} \underbrace{x^{\prime} x} x^{\prime} \stackrel{\text { (1.3) }}{=} x^{4} x^{\prime} x^{\prime} \\
\stackrel{\text { (1.7) }}{=} x^{2} \underbrace{x^{2}\left(x^{2}\right)^{\prime}} \stackrel{(1.3)}{=} x^{2}\left(x^{2}\right)^{\prime} x^{2} \\
\stackrel{(1.5)}{=}\left(x^{2}\right)^{\prime \prime}=x^{2} .
\end{gathered}
$$

Therefore $\left(S, \cdot,^{\prime}\right)$ lies in $\mathcal{E}_{2}$, hence in $\mathcal{W}$.

The variety $\mathcal{W}$ has another characterization that was not mentioned in [10].

Lemma 4. Let $S$ be a semigroup. The following are equivalent:

(1) $S$ is an epigroup in $\mathcal{W}$.

(2) For each $c \in S$, the principal left ideal $S c$ is a completely regular subsemigroup.

(3) For each $c \in S$, the principal right ideal $c S$ is a completely regular subsemigroup.

Proof. An element of a semigroup is completely regular if and only if it lies in some subgroup, so the desired equivalences follow from the definition of $\mathcal{W}$. 
In view of Lemma 4, we should also mention the kindred study in 29] of epigroups $S$ in which every local submonoid $e S e$ is completely regular.

\section{Main Results}

The key tool in the proof of Proposition 2(3) was the following unary operation:

$$
x^{*}=(x c)^{\prime} x(c x)^{\prime} .
$$

Indeed, if $\left(S, \cdot{ }^{\prime}\right)$ is completely regular, then $\left(S, \cdot{ }_{c},{ }^{*}\right)$ is an epigroup in the variety $\mathcal{W}$. However, (囵) was introduced in 10] in an ad hoc fashion. To show that it is quite natural, we note that an ideal of an epigroup is a subepigroup [34, Obs. 4]. In particular, for each $c$ in an epigroup $S, S c$ is a subepigroup. Thus for any $x \in S$, the pseudoinverse $(x c)^{\prime}$ must have the form $y c$ for some $y \in S$. This is exactly what (困) does for us.

Lemma 5. Let $S$ be an epigroup and fix $c \in S$. For all $x \in S$,

$$
(x c)^{\prime}=x^{*} c .
$$

Proof. We compute

$$
(x c)^{\prime}=(x c)^{\prime} x c(x c)^{\prime}=(x c)^{\prime}(x c)^{\prime} x c=(x c) x(c x)^{\prime} \cdot c=x^{*} c,
$$

using (1.2), (1.3) and (1.6).

If $\left(S, \cdot{ }^{\prime}\right)$ is an epigroup, we will refer to $\left(S,{ }^{c},{ }^{*}\right)$ as the unary variant of $\left(S, \cdot{ }^{\prime}\right)$ at $c$. Proposition 2(3) states that if $\left(S, \cdot{ }^{\prime}\right) \in \mathcal{E}_{1}$ is completely regular, then $\left(S,{ }{ }_{c},{ }^{*}\right) \in \mathcal{W}$. Our first main result will both improve and extend this. First we must introduce a family of varieties of unary semigroups. For each $n \in \mathbb{N}$, the variety $\mathcal{V}_{n}$ is defined by associativity and the following identities: (1.2), (1.3),

$$
\begin{aligned}
& x y^{n-1} y^{\prime \prime}=x y^{n} \\
& x^{\prime \prime} x^{n-1} y=x^{n} y
\end{aligned}
$$

Setting $y=x$ in, say, (2.2), we see from Lemma 1 that $\mathcal{V}_{n}$ is a variety of epigroups and in particular,

$$
\mathcal{E}_{n} \subseteq \mathcal{V}_{n} \subseteq \mathcal{E}_{n+1}
$$

That every variant of an epigroup is an epigroup is easy to see, but what is not so obvious is what happens to the pseudoinverse operation. Our first main result clarifies this and also the role of the varieties $\mathcal{V}_{n}$.

Theorem 6. Let $\left(S, \cdot,^{\prime}\right)$ be an epigroup. For each $c \in S$, the unary variant $\left(S,{ }_{c},{ }^{*}\right)$ is an epigroup. If $\left(S, \cdot{ }^{\prime}\right) \in \mathcal{V}_{n}$ for some $n>0$, then $\left(S,{ }_{c},{ }^{*}\right) \in \mathcal{V}_{n}$. Therefore for $n \in \mathbb{N}$, the variety $\mathcal{V}_{n}$ is closed under taking variants.

Corollary 7. Let $\left(S, \cdot{ }^{\prime}\right)$ be a completely regular semigroup. For each $c \in S$, the unary variant $\left(S, \cdot{ }_{c},{ }^{*}\right)$ lies in $\mathcal{V}_{1}$.

Example 8. Not every unary semigroup in $\mathcal{V}_{1}$ is a variant of a completely regular semigroup. Using MACE4, we found that the smallest examples have order 4 , and there are three of them up to isomorphism:

\begin{tabular}{c|cccc}
$\cdot$ & 0 & 1 & 2 & 3 \\
\hline 0 & 1 & 1 & 1 & 1 \\
1 & 1 & 1 & 1 & 1 \\
2 & 1 & 1 & 2 & 1 \\
3 & 1 & 1 & 1 & 3
\end{tabular}

\begin{tabular}{c|cccc}
$\cdot$ & 0 & 1 & 2 & 3 \\
\hline 0 & 1 & 1 & 2 & 2 \\
1 & 1 & 1 & 2 & 2 \\
2 & 2 & 2 & 2 & 2 \\
3 & 2 & 2 & 2 & 3
\end{tabular}

\begin{tabular}{c|cccc}
$\cdot$ & 0 & 1 & 2 & 3 \\
\hline 0 & 2 & 2 & 1 & 1 \\
1 & 2 & 2 & 1 & 1 \\
2 & 1 & 1 & 2 & 2 \\
3 & 1 & 1 & 2 & 3
\end{tabular}

The corresponding pseudoinverse operation is the same for all three epigroups: $0^{\prime}=1$ and $x^{\prime}=x$ for $x=1,2,3$.

Remark 9. For an element $c$ of a semigroup $S$, the mapping $\rho_{c}: S \rightarrow S c ; x \mapsto x c$ is a homomorphism from the variant $\left(S,{ }_{c}\right)$ to $(S c, \cdot)$ since $\left(x \cdot_{c} y\right) c=x c \cdot y c$. If $S$ is also an epigroup, then as already noted, so is $S c$. Every semigroup homomorphism between epigroups is an epigroup homomorphism, but (2.1) shows more explicitly how $\rho_{c}$ preserves pseudoinverses.

Comparing Proposition 2(3) with Corollary 7 raises the question of how the varieties $\mathcal{V}_{1}$ and $\mathcal{W}$ are related other than just the fact that both contain $\mathcal{E}_{1}$. Our second main result addresses this and its corollary connects this discussion to the transitivity of $\sim_{p}$. 


\section{Theorem 10.}

(2) If $\left(S, \cdot,^{\prime}\right)$ is an epigroup in $\mathcal{W}$, then for each $c \in S$, the unary variant $\left(S, \cdot_{c},{ }^{*}\right)$ lies in $\mathcal{V}_{1}$.

In particular, every variant of an epigroup in $\mathcal{W}$ actually lies in a proper subvariety of $\mathcal{W}$, a stronger statement than the assertion that $\mathcal{W}$ is closed under taking variants.

We can now give an affirmative answer to Problem 6.18 in [10. Since $\sim_{p}$ is transitive in $\mathcal{W}$, the theorem immediately implies the following.

Corollary 11. Primary conjugacy $\sim_{p}$ is transitive in every variant of any epigroup in $\mathcal{W}$.

The proofs of Theorems 6 and 10 will be given in 93 In 4 we conclude with some open problems.

\section{Proofs}

Let $\left(S, \cdot,^{\prime}\right)$ be an epigroup and fix $c \in S$. To verify Theorem [6, we start with a few lemmas.

Lemma 12. $\left(S, \cdot{ }_{c},{ }^{*}\right)$ satisfies (1.2) and (1.3).

Proof. First we compute

$$
\begin{aligned}
& x^{*} \cdot{ }_{c} x \cdot{ }_{c} x^{*}=(x c)^{\prime} \underbrace{x(c x)^{\prime}} c x c(x c)^{\prime} \underbrace{x(c x)^{\prime}} \stackrel{(1.6)}{=}(x c)^{\prime} \underbrace{(x c)^{\prime} x c} \underbrace{x c(x c)^{\prime}}(x c)^{\prime} x \\
& \stackrel{(1.3)}{=}(x c)^{\prime} x c(x c)^{\prime} \cdot(x c)^{\prime} x c(x c)^{\prime} \cdot x \stackrel{[1.2]}{=}(x c)^{\prime} \underbrace{(x c)^{\prime} x} \\
& \stackrel{(1.6)}{=}(x c)^{\prime} x(c x)^{\prime}=x^{*},
\end{aligned}
$$

which establishes (1.2).

Next we have

$$
\begin{aligned}
x \cdot{ }_{c} x^{*} & =\underbrace{x c(x c)^{\prime}} x(c x)^{\prime} \stackrel{[1.3)}{=}(x c)^{\prime} x c \underbrace{x(c x)^{\prime}} \\
& \stackrel{(1.6)}{=}(x c)^{\prime} x \underbrace{c(x c)^{\prime}} x \stackrel{(1.6)}{=}(x c)^{\prime} x(c x)^{\prime} c x \\
& =x^{*}{ }_{c} x
\end{aligned}
$$

which establishes (1.3).

We will denote powers of elements in $\left(S,{ }_{c}\right)$ with parentheses in the exponent, that is, $x^{(1)}=x$ and $x^{(n)}=x \cdot{ }_{c} x^{(n-1)}$ for $n>1$.

Lemma 13. If ca has index $n$ in $\left(S, \cdot,^{\prime}\right)$, then a is an epigroup element of index $n$ or $n+1$ in $\left(S, \cdot{ }_{c},{ }^{*}\right)$.

Proof. For any $k>0$, we have $a^{(k+1)}{ }_{c} a^{*}=(a c)^{k+1} a^{*}=a(c a)^{k} \underbrace{c a^{*}}=a \underbrace{(c a)^{k}(c a)^{\prime}}$ using (2.1), and $a^{(k)}=a(c a)^{k}$. Thus if $c a$ has index $n$, then $a^{(n+2)}{ }_{c} a^{*}=a^{(n+1)}$, thus $a$ is an epigroup element of index at most $n+1$ in $\left(S,{ }_{c},{ }^{*}\right)$. If $a$ has index $k \leq n+1$ in $\left(S,{ }_{c},{ }^{*}\right)$, then $a(c a)^{k}(c a)^{\prime}=a(c a)^{k}$ and so $(c a)^{k+1}(c a)^{\prime}=(c a)^{k}$ and so $k \geq n$.

Lemma 14. For all $x \in S$,

$$
c x^{* *}=(c x)^{\prime \prime} .
$$

Proof. Using (荬), we compute

$$
\begin{aligned}
c x^{* *} & =c\left(x^{*}\right) * \quad=\underbrace{c\left(x^{*} c\right)^{\prime}} x^{*}\left(c x^{*}\right)^{\prime} \\
& \stackrel{[1.6]}{=}\left(c x^{*}\right)^{\prime} c x^{*}\left(c x^{*}\right)^{\prime} \stackrel{[1.2)}{=}\left(c x^{*}\right)^{\prime} \\
& =(\underbrace{c(x c)^{\prime}} x(c x)^{\prime})^{\prime} \stackrel{[1.6]}{=}\left((c x)^{\prime} c x(c x)^{\prime}\right)^{\prime(1.2)}=(c x)^{\prime \prime} .
\end{aligned}
$$


Proof of Theorem [6. Assume $\left(S, \cdot,^{\prime}\right)$ is an epigroup. Then by Lemmas 12 and 13 $\left(S,{ }_{c},{ }^{*}\right)$ is also an epigroup.

Suppose now that for some $n \in \mathbb{N},\left(S, \cdot,^{\prime}\right) \in \mathcal{V}_{n}$. Then for all $x, y \in S$,

$$
\begin{aligned}
x \cdot{ }_{c} y^{(n-1)}{ }_{c} y^{* *} & =x(c y)^{n-1} c y^{* *} \stackrel{\text { (3.1) }}{=} x(c y)^{n-1}(c y)^{\prime \prime} \\
& =x(c y)^{n} \quad=x \cdot{ }_{c} y^{(n)},
\end{aligned}
$$

using $\left(S, \cdot{ }^{\prime}\right) \in \mathcal{V}_{n}$ in the third equality. Thus $\left(S, \cdot{ }^{c},{ }^{*}\right) \in \mathcal{V}_{n}$. This completes the proof.

Now we turn to Theorem 10 .

Lemma 15. $\mathcal{V}_{1} \subset \mathcal{W}$

Proof. Fix $\left(S, \cdot{ }^{\prime}\right) \in \mathcal{V}_{1}$. We already know that $S$ is an epigroup in $\mathcal{E}_{2}$ by (2.4) and so by Proposition $2(1)$, we just need to verify the identity $(x y)^{\prime \prime}=x y$. We compute

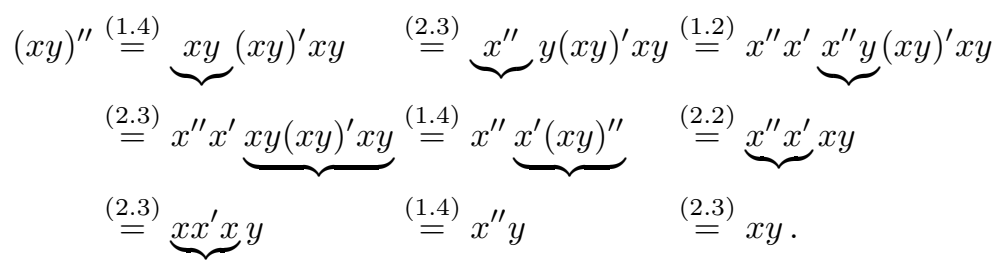

To see that the inclusion is proper, consider the unary semigroup given by the multiplication table

\begin{tabular}{c|cccc}
$\cdot$ & 0 & 1 & 2 & 3 \\
\hline 0 & 2 & 3 & 2 & 2 \\
1 & 1 & 1 & 1 & 1 \\
2 & 2 & 2 & 2 & 2 \\
3 & 3 & 3 & 3 & 3
\end{tabular}

and the unary operation $0^{\prime}=2,1^{\prime}=1,2^{\prime}=2,3^{\prime}=3$. This is easily checked to be an epigroup in $\mathcal{W}$ with ${ }^{\prime}$ as the pseudoinverse operation, but $0^{\prime \prime} \cdot 1=2 \cdot 1=2 \neq 3=0 \cdot 1$, so (2.3) does not hold.

Proof of Theorem 10. Lemma 15 takes care of (1), so we need to prove (2).

Let $\left(S, \cdot{ }^{\prime}\right)$ be an epigroup in $\mathcal{W}$ and fix $c \in S$. Since $\mathcal{W} \subseteq \mathcal{E}_{2} \subseteq \mathcal{V}_{2}$ (by Proposition 2(1) and (2.4)), we know that the unary variant $\left(S,{ }_{c},{ }^{*}\right)$ is an epigroup in $\mathcal{V}_{2}$ (Theorem [6). What remains is to prove that $\left(S,{ }_{c},{ }^{*}\right)$ satisfies (2.2) and (2.3) with $n=1$. We compute

$$
x \cdot{ }_{c} y^{* *}=x c y^{* *} \stackrel{(3.1)}{=} x(c y)^{\prime \prime} \stackrel{(2.2)}{=} x c y=x \cdot{ }_{c} y .
$$

This establishes (2.2) in $\left(S,{ }_{c},{ }^{*}\right)$ and the proof of (2.3) is similar.

\section{Problems}

Completely regular semigroups can be defined conceptually (unions of groups) or as unary semigroups satisfying certain identities. The same is true of the variety $\mathcal{W}$; the conceptual definition given in [10 is that $S$ lies in $\mathcal{W}$ if $S^{2}$ is completely regular or $\mathcal{W}$ can be defined as a variety of unary semigroups (Lemma 3 ).

On the other hand, the epigroup varieties $\mathcal{V}_{n}$ only have a definition as unary semigroups. Since they are closed under taking variants (Theorem [6), they are clearly interesting varieties interlacing the varieties $\mathcal{E}_{n}$ (see (2.4)). Thus one might ask the following.

Problem 1. Is there a conceptual characterization of the varieties $\mathcal{V}_{n}$, or even just $\mathcal{V}_{1}$, analogous to the characterizations of $\mathcal{E}_{1}$ and $\mathcal{W}$ ?

From (2.4) and Theorem 10, we have the following chain of varieties:

$$
\mathcal{E}_{1} \subset \mathcal{V}_{1} \subset \mathcal{W} \subset \mathcal{E}_{2} \subset \mathcal{V}_{2} \subset \mathcal{E}_{3} \cdots
$$

Problem 2. Is there a natural family of varieties $\mathcal{W}_{n}$ interlacing the varieties in the chain above and such that $\mathcal{W}_{1}=\mathcal{W}$ ? In addition, does the appropriate generalization of Theorem 10(2) hold? 
An interesting direction for the study of the varieties $\mathcal{V}_{n}$ or $\mathcal{W}$ is to consider the subvarieties in which idempotents commute, that is, so-called $E$-semigroups (see [1] and the references therein). These are subvarieties because every idempotent in an epigroup has the form $x^{\prime} x$, and so $E$-epigroups are characterized by the identity $x^{\prime} x y^{\prime} y=y^{\prime} y x^{\prime} x$.

Problem 3. Study the varieties of E-epigroups in $\mathcal{V}_{n}$ or $\mathcal{W}$.

Many classes of algebras can be characterized by forbidden subalgebras or forbidden divisors (quotients). For example, distributive lattices can be characterized by two forbidden sublattices; similarly, stable semigroups can be characterized by forbidding the bicyclic monoid as a subsemigroup [28]; see also [9] for another example. The considerations in the paper prompt the following natural problems.

Problem 4. Can any of the inclusions of varieties considered here, especially $\mathcal{E}_{1} \subset \mathcal{V}_{1}$ and $\mathcal{V}_{1} \subset \mathcal{W}$, be characterized by forbidden subepigroups or forbidden epidivisors?

Finally, returning to primary conjugacy, we rephrase two problems from $[10$ to the context of this paper. These were suggested to us by Prof. J. Araújo.

Problem 5. Characterize and enumerate primary conjugacy classes in various types of transformation semigroups and their variants such as, for example, those appearing in the problem list of [13, §6] or those appearing in the list of transformation semigroups included in [17]. Especially interesting would be a characterization of primary conjugacy classes in variants of centralizers of idempotents [2, 11, 12, or in variants of semigroups in which the group of units has a rich structure [3, 5, 6, 7, 8, 14.

In [4, a problem on independence algebras was solved using their classification; the same technique might perhaps be used to extend the results in [15] and to solve the following.

Problem 6. Characterize $\sim_{p}$ in the variants of the endomorphism monoid of a finite dimensional independence algebra.

\section{ACKNOWLEDGEMENT}

This paper is part of the first author's dissertation for the PhD Program in Computational Algebra at Universidade Aberta in Portugal. Most of the proofs were obtained with the assistance of the automated theorem prover Prover9 developed by McCune [31.

\section{REFERENCES}

[1] J. Almeida, J.-E. Pin and P. Weil, Semigroups whose idempotents form a subsemigroup, Math. Proc. Camb. Phi. Soc. 111 (1992), 241-253.

[2] J.M. André, J. Araújo and J. Konieczny, Regular centralizers of idempotent transformations, Semigroup Forum 82 (2) (2011), 307-318.

[3] J.M. André, J. Araújo and P.J. Cameron, The classification of partition homogeneous groups with applications to semigroup theory, J. Algebra 452 (2016), 288310.

[4] J. Araújo, W. Bentz and J. Konieczny, The largest subsemilattices of the endomorphism monoid of an independence algebra, Linear Algebra Appl. 458 (2014), 60-79.

[5] J. Araújo, W. Bentz, J.D. Mitchell and C. Schneider, The rank of the semigroup of transformations stabilising a partition of a finite set, Math. Proc. Cambridge Philos. Soc. 159 (2015), no. 2, 339-353.

[6] J. Araújo and P. J. Cameron, Two generalizations of homogeneity in groups with applications to regular semigroups, Trans. Amer. Math. Soc. 368 (2016), no. 2, 1159-1188.

[7] J. Araújo and P. J. Cameron, Primitive groups synchronize non-uniform maps of extreme ranks, J. Combin. Theory Ser. B 106 (2014), 98-114.

[8] J. Araújo, P.J. Cameron, J.D. Mitchell and M. Neunhöffer, The classification of normalizing groups, J. Algebra, 373 (2013), 481-490.

[9] J. Araújo, M. Kinyon and A. Malheiro, A characterization of adequate semigroups by forbidden subsemigroups, Proc. Roy. Soc. Edinburgh Sect. A 143 (2013), no. 6, 1115-1122.

[10] J. Araújo, M. Kinyon, J. Konieczny and A. Malheiro, Four notions of conjugacy for abstract semigroups, Proc. Roy. Soc. Edinburgh Sect. A 147 (2017), no. 6, 1169-1214.

[11] J. Araújo and J. Konieczny, Automorphisms groups of centralizers of idempotents, J. Algebra 269 (2003), $227-239$.

[12] J. Araújo and J. Konieczny, Semigroups of transformations preserving an equivalence relation and a cross-section, Comm. Algebra 32 (2004), 1917-1935.

[13] J. Araújo and J. Konieczny, Centralizers in the full transformation semigroup, Semigroup Forum 86 (2013), 1-31.

[14] J. Araújo and C. Schneider, The rank of the endomorphism monoid of a uniform partition, Semigroup Forum 783 (2009), $498-510$ 
[15] I. Dolinka and J. East, Semigroups of rectangular matrices under a sandwich operation, Semigroup Forum 96 (2018), no. $2,253-300$.

[16] I. Dolinka and J. East, Variants of finite full transformation semigroups, Internat. J. Algebra Comput. 25 (2015), no. 8, $1187-1222$.

[17] V.H. Fernandes, Presentations for some monoids of partial transformations on a finite chain: a survey, Semigroups, algorithms, automata and languages (Coimbra, 2001), 363-378, World Sci. Publ., River Edge, NJ, 2002.

[18] O. Ganyushkin and V. Mazorchuk, Classical finite transformation semigroups, Algebra and Applications 9, Springer-Verlag London, Ltd., London, 2009.

[19] P.M. Higgins, The semigroup of conjugates of a word, Internat. J. Algebra Comput. 16 (2006), 1015-1029.

[20] J. B. Hickey, On variants of a semigroup, Bull. Austral. Math. Soc. 34 (1986), no. 3, 447-459.

[21] J.M. Howie, Fundamentals of semigroup theory, London Mathematical Society Monographs, Clarendon Press, Oxford, 1995.

[22] T.A. Khan and M.V. Lawson, Variants of regular semigroups, Semigroup Forum 62 (2001), no. 3, 358-374.

[23] G. Kudryavtseva, On conjugacy in regular epigroups, arXiv.org:0605698

[24] G. Kudryavtseva and V. Maltcev, On representations of variants of semigroups, Bull. Austral. Math. Soc. 73 (2006), no. $2,273-283$.

[25] G. Kudryavtseva and V. Mazorchuk, On conjugation in some transformation and Brauer-type semigroups, Publ. Math. Debrecen 70 (2007), 19-43.

[26] G. Kudryavtseva and V. Mazorchuk, On three approaches to conjugacy in semigroups, Semigroup Forum 78 (2009), 14-20.

[27] R. Jongchotinon, S. Chaopraknoi and Y. Kemprasit, Isomorphism theorems for variants of semigroups of linear transformations, Int. J. Algebra 4 (2010), no. 25-28, 1407-1412.

[28] G. Lallement, Semigroups and Combinatorial Applications, John Wiley \& Sons, New York, 1979.

[29] J. Liu, Q. Chen and C. Han, Locally completely regular epigroups, Comm. Algebra 44 (2016), 4546-4563.

[30] V. Mazorchuk and G. Tsyaputa, Isolated subsemigroups in the variants of $T_{n}$, Acta Math. Univ. Comenian. 77 (2008), no. $1,63-84$.

[31] W. McCune, Prover9 and Mace4, https://www.cs.unm.edu/ mccune/prover9/

[32] H. Pei, L. Sun and H. Zhai, Green's relations for the variants of transformation semigroups preserving an equivalence relation, Comm. Algebra 35 (2007), no. 6, 1971-1986.

[33] M. Petrich and N.R. Reilly, Completely Regular Semigroups, John Wiley \& Sons, New York, 1999.

[34] L. N. Shevrin, On the theory of epigroups. I. (Russian) Mat. Sb. 185 (1994), 129-160; translation in Russian Acad. Sci. Sb. Math. 82 (1995), 485-512

[35] L. N. Shevrin, Epigroups, in V. B. Kudryavtsev and I. G. Rosenberg (eds.), Structural Theory of Automata, Semigroups, and Universal Algebra, 331-380, NATO Sci. Ser. II Math. Phys. Chem., 207, Springer, Dordrecht, 2005.

[36] G. Tsyaputa, Isolated and nilpotent subsemigroups in the variants of $\mathcal{I S}_{n}$, Algebra Discrete Math. 2006, 89-97.

(Borralho) Universidade Aberta, R. Escola Politécnica, 147, 1269-001 Lisboa, Portugal

(Borralho) Cemat-CiênCias, Departamento de Matemática, Faculdade de CiÊncias, Universidade de Lisboa, 1749-016, Lisboa, Portugal

(Kinyon) Department of Mathematics, University of Denver, Denver, CO 80208, USA

(Kinyon) Centre for Mathematics and Applications, Faculdade de Ciências e Tecnologia, Universidade Nova de Lisboa, Campus da Caparica, 2829-516 Caparica, PT

E-mail address, Borralho: mfborralho@gmail.com

E-mail address, Kinyon: mkinyon@du.edu 